\title{
Determining the prices of the medicines in the absence of superiority over alternative medical technology
}

\author{
Wojciech Matusewicz, Katarzyna Jagodzińska-Kalinowska, Aneta Lipińska, Maciej Pomorski*
}

From 3rd International PPRI Conference 2015: Pharmaceutical Pricing and Reimbursement Policies: Challenges Beyond the Financial Crisis

Vienna, Austria. 12-13 October 2015

\section{Problem statement}

When there are no RCTs which prove that the new technology is better than the current drug or non-drug medical technologies currently financed by the public payer, the MAH is obliged to use the price equal to the cheapest reimbursed alternative.

\section{Objectives}

Presentation of a method offered by the new Reimbursement Act (RA), which allows one to determine the prices of medicines in the absence of superiority over alternative medical technology. This price is calculated by the AHTATS and must be placed in the Recommendation of the President of the AHTATS.

\section{Methods}

The primary goal of the new RA is to implement the principle of economical production. This should be kept in mind, while interpreting its content, because the act allows one to approach to each drug individually.

At the beginning one needs to find a comparator currently financed with the best outcomes/cost ratio. The next step is to search for RCTs proving the superiority of the proposed drug over the designated comparator. In cases, where there are no such RCTs in the MAH's submission, the official sales price of the drug, must be calculated in such a way that the cost of using it would not be higher than using the designated comparator. This has significant consequences because the Ministry of Health is obliged to use the calculated price in its final reimbursement decision.

\footnotetext{
* Correspondence: m.pomorski@aotm.gov.pl

Agency for Health Technology Assessment and Tariff System (AHTATS), Warsaw, 02-611, Poland
} 